\title{
Investigations on Deer to Deer and Deer to Cattle Transmission of the Vaccine Mycobacterium bovis Bacillus Calmette-Guérin (BCG)
}

\author{
Mitchell V. Palmer ${ }^{1 *}$, Tyler C. Thacker ${ }^{1}$, W. Ray Waters ${ }^{1}$, Suelee Robbe-Austerman ${ }^{2}$ and Beth N. Harris ${ }^{2}$ \\ ${ }^{1}$ United States Department of Agriculture, Agricultural Research Service, National Animal Disease Center, 1920 Dayton Avenue, Ames, IA, 50010, USA \\ ${ }^{2}$ United States Department of Agriculture, Veterinary Services, National Veterinary Services Laboratories, 1800 Dayton Avenue, Ames, IA, 50010, USA
}

\begin{abstract}
Mycobacterium bovis is the causative agent of tuberculosis in animals and can cause tuberculosis in humans clinically indistinguishable from that caused by Mycobacterium tuberculosis. Efforts to eradicate bovine tuberculosis have significantly decreased prevalence in developed countries. However, some countries have found it impossible to eradicate bovine tuberculosis due to the presence of a wildlife reservoir of $M$. bovis. In Michigan, USA there exists a reservoir of $M$. bovis in free-ranging white-tailed deer. Vaccination with $M$. bovis BCG is one approach to controlling tuberculosis in wildlife. Nevertheless, use of a live vaccine generates concerns about exposure risk to non-target species, including domestic livestock. Unintentional exposure of cattle to BCG may result in increased numbers of false positive tuberculin skin test reactions. Twenty-nine white-tailed deer received $1 \mathrm{SC}$ dose of $10^{7}$ colony-forming units of $M$. bovis BCG Danish $1331(n=19)$ or no vaccination $(n=10)$. Vaccinated and non-vaccinated deer were comingled with opportunity for direct and indirect contact. Twelve unvaccinated Holstein calves were housed in a separate paddock with no means of direct contact with deer; however, indirect contact through the sharing of feed and water was permitted. After 180 days, 11 out of 15 vaccinated deer and 4 out of 8 non-vaccinated deer were classified as reactors using the tuberculin skin test. All 12 calves were categorized as non-reactors, by both the tuberculin skin test and BOVIGAM ${ }^{\mathrm{TM}}$ assay. Vaccination of free-ranging white-tailed deer with BCG Danish is unlikely to have a deleterious effect on tuberculosis surveillance measures in cattle.
\end{abstract}

Keywords: Deer; Mycobacteria; Tuberculosis; Vaccination; Wildlife

\section{Introduction}

Mycobacterium bovis is the causative agent of tuberculosis in animals and has one of the broadest host ranges of any pathogen. Mycobacterium bovis can cause tuberculosis in humans clinically indistinguishable from that caused by Mycobacterium tuberculosis. In the early $20^{\text {th }}$ century, public health concerns posed by transmission of $M$. bovis from cattle to humans, through consumption of contaminated milk or meat, prompted the United States, and other countries, to implement national programs to eradicate tuberculosis from cattle. Efforts have centered on slaughter of cattle reacting positively to a tuberculin skin test, and most eradication campaigns have been successful in decreasing the prevalence of bovine tuberculosis. However, some countries have found it impossible to eradicate bovine tuberculosis due to the presence of a wildlife reservoir of $M$. bovis. In most cases, wildlife originally acquired tuberculosis from cattle; however, the disease is now spilling back from wildlife to cattle, impeding the progress of eradication $[1,2]$. In an effort to reduce wildlife to cattle transmission of $M$. bovis some countries are investigating the possible role of wildlife vaccination.

In 1994, a free-ranging white-tailed deer (Odocoileus virginianus) in Michigan, USA was diagnosed with tuberculosis due to M. bovis [3]. Subsequent surveys identified the first known reservoir of $M$. bovis in free-ranging wildlife in the United States and a significant impediment to ongoing efforts to eradicate bovine tuberculosis from domestic livestock [4,5]. Current disease control measures include, among other things, decreasing deer density through increased hunting. Control and surveillance measures have now been in place in Michigan for over 10 years and a significant reduction in apparent prevalence of tuberculosis in deer has been achieved; however, public support for further population reduction is waning [6]. Vaccination of deer could be used in areas of sustained high disease prevalence, in order to prevent infection, disease, or transmission.
Recently, protection to experimental infection with $M$. bovis, was demonstrated by subcutaneous (SC) and oral vaccination of whitetailed deer with M. bovis Bacillus Calmette Guerin (BCG) Danish $[7,8]$. However, transmission of BCG from vaccinated deer to co-mingled unvaccinated deer was also documented [7].

Potential vaccination of wildlife with a live vaccine generates various concerns, not least of which is the exposure risk to nontarget species, including domestic livestock. The purpose of this study was to evaluate the potential for transmission of BCG from vaccinated deer to unvaccinated deer or cattle through direct or indirect contact, respectively. Unintentional exposure of cattle to BCG may result in increased numbers of false-positive tuberculin skin test reactions. An increase in false-positive reactions would confound current bovine tuberculosis surveillance efforts and lead to the unnecessary slaughter of otherwise normal animals.

\section{Materials and methods}

\section{Animals and vaccination}

Twenty-nine white-tailed deer (6-18 months old) were obtained

*Corresponding author: Mitchell V. Palmer, United States Department of Agriculture, Agricultural Research Service, National Animal Disease Center, 1920 Dayton Avenue, Ames, IA, 50010, USA, Tel: 515-337-7474; Fax: 515-337-7428; E-mail: mitchell.palmer@ars.usda.gov

Received October 14, 2010; Accepted November 10, 2010; Published November 12, 2010

Citation: Palmer MV, Thacker TC, Waters WR, Robbe-Austerman S, Harris BN (2010) Investigations on Deer to Deer and Deer to Cattle Transmission of the Vaccine Mycobacterium bovis Bacillus Calmette-Guérin (BCG). J Vaccines Vaccin 1: 104. doi:10.4172/2157-7560.1000104

Copyright: (c) 2010 Palmer MV, et al. This is an open-access article distributed under the terms of the Creative Commons Attribution License, which permits unrestricted use, distribution, and reproduction in any medium, provided the original author and source are credited. 
from a captive breeding herd (tuberculosis free) at the National Animal Disease Center (Ames, lowa, USA). All deer were housed and cared for according to institutional guidelines. Deer were randomly assigned to one of two groups, $1 \mathrm{SC}$ dose of $10^{7}$ colony-forming units (CFU) $M$. bovis BCG Danish $1331(\mathrm{n}=19)$ or no vaccination $(\mathrm{n}=10)$. Deer were vaccinated subcutaneously on the right side of the neck, midway between the head and shoulder. Vaccinated and non-vaccinated deer were housed together in an outdoor paddock with shared feeding and watering sites. Twelve Holstein calves ( 6 months old, castrated males) were not vaccinated and housed in a similar, but separate, paddock with no means of direct contact with deer. On a daily basis, deer were moved to the cattle paddock and cattle were moved to the deer paddock in such a manner as to prevent direct deer-cattle contact. Deer feeding was done such that excess feed remained in feeders when cattle entered the deer paddock. Switching of pens to afford an opportunity for indirect deer-cattle contact proceeded for 180 days.

\section{Vaccine}

The $M$. bovis BCG Danish 1331 strain was grown in Middlebrook's 7H9 media supplemented with $10 \%$ oleic acid-albumin-dextrose complex (Difco, Detroit, MI) plus 0.05\% Tween 80 (Sigma Chemical Co., St. Louis, MO) as described for virulent M. bovis [9]. Mid logphase growth bacilli were pelleted by centrifugation at $750 \mathrm{x} g$, washed twice with phosphate buffered saline (PBS) $(0.01 \mathrm{M}, \mathrm{pH} 7.2)$, and diluted to the appropriate cell density in $2 \mathrm{ml}$ of PBS. Bacilli were enumerated by serial dilution plate counting on Middlebrook's 7H11 selective media (Becton Dickinson, Cockeysville, MD). A single vaccine dose consisted of $10^{7} \mathrm{CFU} M$. bovis BCG in $2.0 \mathrm{ml}$ PBS.

\section{Evaluation of vaccine shedding}

Immediately prior to the study, and again at the conclusion of the study, all deer and calves were tested for exposure to $M$. bovis by use of the comparative cervical tuberculin skin test (CCT) as described previously $[10,11]$. Deer and calves were categorized as reactors or non-reactors, according to USDA guidelines [12]. At the same time, cattle were also evaluated using a commercially available interferongamma (IFN- $\gamma$ ) assay (BOVIGAM ${ }^{\mathrm{TM}}$, Prionics, Zurich, Switzerland) as described previously [10]. Both the CCT and the BOVIGAM ${ }^{\mathrm{TM}}$ assay are approved tests for cattle in the United States Department of Agriculture (USDA) bovine tuberculosis eradication effort. The USDA has also approved the CCT for use in deer. The CCT requires injection of purified protein derivative (PPD) from $M$. bovis (National Veterinary Services Laboratories, USDA, Ames, IA) and Mycobacterium avium (National Veterinary Services Laboratories) at 2 different sites on the lateral neck [12]. Exposure to M. bovis is indicated when the change in skin thickness at the M. bovis PPD injection site is greater than that measured at the $M$. avium PPD injection site. Conversely, exposure to non-tuberculous mycobacteria is indicated by a change in skin thickness at the $M$. avium injection site greater than that measured at the M. bovis PPD injection site [12].

All deer were monitored for vaccine shedding by collection of oronasal swabs and feces. Samples were collected prior to vaccination and on days $14,28,42,56,89$ and 179 after vaccination. One swab from each nostril and one oral swab were processed together representing a single oronasal sample from each deer at each time point. Approximately $2 \mathrm{gm}$ feces was obtained from each deer at each time point and processed for isolation of $M$. bovis BCG.

\section{Necropsy and tissue processing}

All deer and all calves were euthanized by intravenous sodium pentobarbital and examined at the termination of the study (180 days after vaccination). At necropsy, the following tissues were examined grossly; liver, lung; mandibular, parotid, medial retropharyngeal, tracheobronchial, mediastinal, hepatic, mesenteric and superficial cervical lymph nodes; and palatine tonsil. Samples of mandibular, parotid, medial retropharyngeal, tracheobronchial, mediastinal, hepatic and mesenteric lymph nodes were collected for bacteriological isolation of BCG as described [13]. Tissues from an individual deer were submitted for bacteriological culture in 3 pools; head pool (medial retropharyngeal, mandibular and parotid lymph nodes), thoracic pool (tracheobronchial and mediastinal lymph nodes) and abdominal pool (mesenteric and hepatic lymph nodes). Tissues with gross lesions compatible with tuberculosis were further processed for microscopic analysis as described $[14,15]$.

\section{Isolation and identification of mycobacterial isolates}

Tissue and swab samples were processed for isolation of $M$. bovis BCG as previously described [13] using both the BACTEC 460 radiometric system and BACTEC Mycobacteria Growth Indicator Tube (MGIT) 960 system (Becton Dickinson and Company, Sparks, MD). Isolates of $M$. bovis BCG were identified by a combination of ZiehlNeelsen acid-fast staining, biochemical tests and nucleic acid probes (ACCUPROBE, Gen-Probe, San Diego, CA). Further identification was done using $16 \mathrm{~S}$ ribosomal DNA sequencing as described previously [16]. Sequences were then identified through use of a mycobacterial species sequence database [17].

From some oronasal swabs a mycobacterial species was isolated; however, identification by the above methods was not possible due to contamination and overgrowth by non-mycobacteria. These mycobacterial isolates were further analyzed by PCR using the following protocol. Two milliliters of MGIT culture were centrifuged for $3 \mathrm{~min}$ at $10,000 \times \mathrm{g}$ to pellet mycobacteria. Pellets were washed with $1 \mathrm{ml}$ Tris-EDTA (TE) buffer. The pellet was suspended in 200 $\mu \mathrm{l}$ Buffer AL (Qiagen, Valencia, CA) and lysed by boiling for $8 \mathrm{~min}$. After cooling to less than $56^{\circ} \mathrm{C}, 20 \mu$ l Proteinase $\mathrm{K}$ (Qiagen) was added and incubated overnight at $56^{\circ} \mathrm{C}$ or until completely lysed. Liberated DNA was isolated using QIAMP DNA Mini Kit (Qiagen) according to the manufacturer's directions. Detection of IS6110 was performed in $50 \mu \mathrm{l}$ reactions using FASTSTART Taq (Roche Applied Science, Indianapolis, IN) according to manufacturer's directions using primers for IS6110 as described previously [18]. Duplicate reactions were run using primers Sp1 and Sp2 [19] to serve as a PCR positive control and to confirm the presence of mycobacteria. Samples were analyzed by electrophoresis on $1.5 \%$ agarose gel and visualized with ethidium bromide.

\section{Results}

\section{Tuberculin skin testing and BOVIGAM ${ }^{\mathrm{TM}}$}

Prior to the study all deer and all calves were classified as nonreactors (negative for exposure to $M$. bovis) according to USDA guidelines for interpretation of CCT results [12]. Likewise all calves were categorized as negative using the BOVIGAM $^{\mathrm{TM}}$ assay and manufacturer's recommendations for interpretation of results. During the course of the study 6 deer died of causes unrelated to the experimental procedure (trauma, necrobacillosis, or Pasteurella multocida induced pneumonia). Two of six were non-vaccinated deer and 4 of 6 were vaccinated deer. From these 6 deer, tissues were collected and processed as described above.

At the conclusion of the study ( $\mathrm{n}=23$ deer) 11 out of 15 vaccinated 
deer and 4 out of 8 non-vaccinated deer were classified as reactors using the CCT. All other deer were categorized as non-reactors (Table 1). All 12 calves were categorized as non-reactors or negative, at the conclusion of the study, by both the CCT and BOVIGAM ${ }^{\mathrm{TM}}$ assay, respectively.

\section{Vaccine shedding}

From vaccinated and non-vaccinated deer, a total of 160 oronasal swabs and 150 fecal specimens were processed for bacteriological culture. At no time during the course of the study was BCG isolated from fecal samples. Bacteriological culture of oronasal samples yielded a number of isolates of non-tuberculous mycobacteria. Mycobacterium fortuitum was isolated from 5 of 19 vaccinated deer (Table 2) and 9 of 10 non-vaccinated deer (Table 1). Isolations were from a single time point from 9 out of the 14 deer and multiple time points from 5 out of the 14 deer (Table 1 and Table 2). Mycobacterium smegmatis was isolated from oronasal swabs from 2 vaccinated deer at one time point each and Mycobacterium alvei was isolated from 2 non-vaccinated deer at a single time point. From 84 of the 160 oronasal samples processed, an acid fast bacillus belonging to the genus Mycobacteria was isolated, but further speciation was prohibited due to contamination and overgrowth by nonmycobacteria. PCR analysis of these isolates identified 2 out of 84 as BCG; isolated from 1 vaccinated deer 89 days after vaccination and 1 non-vaccinated deer 56 days after vaccination (Table 1 and Table 2). Final CCT results identified the vaccinated deer as a reactor and the non-vaccinated deer as a non-reactor.

\section{Necropsy and tissue processing}

Focal caseonecrotic granulomas $(1-1.5 \mathrm{~cm})$ were seen in the medial retropharyngeal lymph nodes of 1 vaccinated deer (skin test reactor) and 1 non-vaccinated deer (skin test non-reactor). Microscopically, lesions were characterized by coalescent caseonecrotic granulomas composed of macrophages, lymphocytes and multinucleated giant cells, all surrounded by thin bands of collagen fibers. Ziehl-Neelsen staining did not reveal acid-fast bacteria in any lesions. BCG was isolated from tissue samples of 6 out of 19 vaccinated deer and 1 out of 10 non-vaccinated deer. Specifically BCG was isolated from tissues of the thoracic pool from each of the 6 vaccinated deer and the single non-vaccinated deer. Additionally, BCG was isolated from tissues of the head pool from the non-vaccinated deer (Table 1). BCG was not isolated from either deer with caseonecrotic granulomatous lesions in the medial retropharyngeal lymph nodes. In addition to BCG, Mycobacterium avium, Mycobacterium kansasii and Mycobacterium simiae were also isolated from a single deer each.

\section{Discussion}

The present study suggests that indirect contact would not result in transmission of BCG Danish from vaccinated deer to cattle, even under circumstances of repeated and sustained indirect contact. If indirect transmission were to occur, the magnitude of shedding would likely be critical. Shedding of BCG was seen inconsistently and in a limited fashion from oronasal samples, and not from feces. In humans with suspected tuberculosis, sputum and gastric fluids may be examined for acid-fast bacilli. Oronasal samples, as collected in the present study, would be most analogous to sputum samples from humans. Samples of rumen or abomasal fluids (analogous to gastric fluid) were not examined in the present study.

Previous studies have shown that oral exposure of calves to BCG does not reliably induce positive tuberculin reactivity as measured by the intradermal skin test or IFN- $\gamma$ assays [20]. Reports of oral vaccination of cattle with $10^{8}$ or $10^{10} \mathrm{CFU}$ BCG demonstrated a positive skin test response in 3 of 9 and 6 of 10 calves, respectively. Four of nineteen orally vaccinated calves developed positive responses to the standard IFN- $\gamma$ assay. In contrast all calves vaccinated SC with $10^{6} \mathrm{CFU}$ of BCG developed positive skin test responses. Clearly, in cattle, route of vaccination and vaccine dose affect tuberculin skin test status. Although the CCT and the BOVIGAM ${ }^{\mathrm{TM}}$ assays may not identify all cattle exposed to BCG, especially those exposed by the oral route; in the current study, bacteriological culture of tissues from non-vaccinated calves also failed to yield BCG, suggesting that BCG was not transmitted and did not colonize cattle.

Natural behavior of deer and cattle will impact the degree of contact, either direct or indirect. In the present study a form of indirect contact through sharing of feed and exposure to soiled paddocks

\begin{tabular}{|c|c|c|c|c|c|c|}
\hline \multirow[b]{2}{*}{ Deer ID } & \multirow[b]{2}{*}{ Pre $\mathrm{CCT}^{1}$} & \multirow[b]{2}{*}{ Post $\mathrm{CCT}^{2}$} & \multicolumn{4}{|c|}{ Bacteriological Culture } \\
\hline & & & Feces $^{3}$ & Oronasal $^{3}$ & Tissue $^{4}$ & Tissue Pool ${ }^{5}$ \\
\hline 13 & Neg & Neg & Neg & BCG $^{6}(56)$ M. fortuitum (14) UIM $(14,28,42,56,89)$ & Neg & NA \\
\hline 35 & Neg & Neg & Neg & M. fortuitum $(28,56,89)$ M. alvei (14) UIM (42) & Neg & NA \\
\hline 1072 & Neg & Neg & Neg & M. fortuitum (179) UIM $(28,42,56,179)$ & Neg & NA \\
\hline $32 \mathrm{M}$ & Neg & Neg & Neg & M. fortuitum (14) UIM $(14,28,56,89)$ & Neg & NA \\
\hline $4 M^{7}$ & Neg & ND & Neg & M. fortuitum (14) UIM $(14,28,42,56)$ & M. simiae & Head \\
\hline $6 \mathrm{M}^{7}$ & $\mathrm{Neg}$ & ND & $\mathrm{Neg}$ & M. fortuitum (14) UIM (42) & M. kansasii & Thoracic \\
\hline $1 \mathrm{M}$ & Neg & Reactor & Neg & UIM $(14,42,56,89)$ & BCG & Head, Thoracic \\
\hline 14 & Neg & Reactor & Neg & M. fortuitum (14) M. alvei $(28)$ UIM $(14,42)$ & Neg & NA \\
\hline 29 & Neg & Reactor & Neg & M. fortuitum (89) UIM $(28,42)$ & Neg & NA \\
\hline $20 \mathrm{M}$ & Neg & Reactor & Neg & M. fortuitum (14) UIM $(14,28,42,56,89,179)$ & Neg & NA \\
\hline
\end{tabular}

${ }^{1}$ Comparative cervical tuberculin skin test (CCT) prior to comingling. Neg = no evidence of prior exposure to $M$. bovis.

${ }^{2} \mathrm{CCT}$ after 180 days of comingling.

${ }^{3}$ Feces and oronasal swab samples collected prior to comingling and on days 14, 28, 42, 56, 89 and 179 days of comingling. Isolate (sampling day after comingling). ${ }^{4}$ Isolation of mycobacteria from tissues collected at necropsy after 180 days of comingling. $\mathrm{Neg}=$ no mycobacteria isolated.

${ }^{5}$ For bacteriological isolation of mycobacteria, tissues were allocated into one of three pools; head (mandibular, parotid and medial retropharyngeal lymph nodes), thoracic (lung, mediastinal and tracheobronchial lymph nodes), or abdominal (mesenteric and hepatic lymph nodes).

${ }^{6}$ Unspeciated mycobacteria later identified by PCR as M. bovis BCG Danish.

${ }^{7}$ Deer died due to trauma unrelated to the experimental protocol prior to end of study.

UIM = Unspeciated mycobacteria not found not to be M. bovis BCG Danish by PCR.

NA $=$ not applicable.

$\mathrm{ND}=$ not done.

Table 1: Summary of intradermal tuberculin test and bacteriological culture results from unvaccinated deer co-mingled with Mycobacterium bovis BCG Danish vaccinated deer for 180 days. 
Citation: Palmer MV, Thacker TC, Waters WR, Robbe-Austerman S, Harris BN (2010) Investigations on Deer to Deer and Deer to Cattle Transmission of the Vaccine Mycobacterium bovis Bacillus Calmette-Guérin (BCG). J Vaccines Vaccin 1: 104. doi:10.4172/2157-7560.1000104

Page 4 of 5

\begin{tabular}{|c|c|c|c|c|c|c|}
\hline & & & \multicolumn{4}{|c|}{ Bacteriological Culture } \\
\hline Deer ID & Pre $\mathrm{CCT}^{1}$ & Post $\mathrm{CCT}^{2}$ & Feces $^{3}$ & Oronasal $^{3}$ & Tissue $^{4}$ & Tissue Pool ${ }^{5}$ \\
\hline 38 & $\mathrm{~N}$ & $\mathrm{~N}$ & $\mathrm{~N}$ & M. fortuitum $(14,28,42,56)$ & Neg & NA \\
\hline $21 \mathrm{M}$ & $\mathrm{N}$ & $\mathrm{N}$ & $\mathrm{N}$ & M. fortuitum (179) UIM $(28,42,89)$ & Neg & NA \\
\hline $23 \mathrm{M}$ & $\mathrm{N}$ & $\mathrm{N}$ & $\mathrm{N}$ & UIM $(14,42,89)$ & Neg & NA \\
\hline $24 \mathrm{M}$ & $\mathrm{N}$ & $\mathrm{N}$ & $\mathrm{N}$ & M. smegmatis (14) UIM $(28,42)$ & BCG & Thoracic, Abdominal \\
\hline 15 & $\mathrm{~N}$ & $\mathrm{R}$ & $\mathrm{N}$ & M. fortuitum (14) UIM $(28,42)$ & Neg & NA \\
\hline 36 & $\mathrm{~N}$ & $\mathrm{R}$ & $\mathrm{N}$ & M. fortuitum (179) UIM $(28,42,56)$ & BCG & Thoracic \\
\hline $28 \mathrm{M}$ & $\mathrm{N}$ & $\mathrm{R}$ & $\mathrm{N}$ & UIM $(14,28,42,56,89)$ & Neg & NA \\
\hline $2 \mathrm{M}$ & $\mathrm{N}$ & $\mathrm{R}$ & $\mathrm{N}$ & UIM $(28,42,56,89)$ & Neg & NA \\
\hline $30 \mathrm{M}$ & $\mathrm{N}$ & $\mathrm{R}$ & $\mathrm{N}$ & UIM $(14,28,42)$ & Neg & NA \\
\hline $34 \mathrm{M}$ & $\mathrm{N}$ & $\mathrm{R}$ & $\mathrm{N}$ & $\mathrm{BCG}^{6}(56)$ UIM $(28,42,56)$ & M. avium & Thoracic \\
\hline $38 \mathrm{M}$ & $\mathrm{N}$ & $\mathrm{R}$ & $\mathrm{N}$ & M. fortuitum $(14,179)$ UIM $(28,42,56)$ & BCG & Thoracic \\
\hline $33 \mathrm{M}$ & $\mathrm{N}$ & $\mathrm{R}$ & $\mathrm{N}$ & M. fortuitum (14) UIM $(28,42,89)$ & Neg & NA \\
\hline $3 \mathrm{M}$ & $\mathrm{N}$ & $\mathrm{R}$ & $\mathrm{N}$ & M. fortuitum (179) UIM $(14,89,179)$ & Neg & NA \\
\hline $5 \mathrm{M}$ & $\mathrm{N}$ & $\mathrm{R}$ & $\mathrm{N}$ & UIM $(14,28,42)$ & Neg & NA \\
\hline $7 \mathrm{M}$ & $\mathrm{N}$ & $\mathrm{R}$ & $\mathrm{N}$ & UIM $(14,28,42,56)$ & Neg & NA \\
\hline $22^{6}$ & $\mathrm{~N}$ & ND & $\mathrm{N}$ & M. fortuitum (14) UIM $(42,89)$ & BCG & Thoracic \\
\hline $28^{7}$ & $\mathrm{~N}$ & ND & $\mathrm{N}$ & UIM $(14,28,42)$ & Neg & NA \\
\hline $19 M^{7}$ & $\mathrm{~N}$ & ND & $\mathrm{N}$ & M. fortuitum $(56)$ UIM $(28,42,89)$ & BCG & Thoracic \\
\hline $18 M^{7}$ & $\mathrm{~N}$ & ND & $\mathrm{N}$ & M. fortuitum (56) UIM $(14,28,42,89)$ & BCG & Thoracic, Abdomen \\
\hline
\end{tabular}

${ }^{1}$ Comparative cervical tuberculin skin test prior to comingling. Neg $=$ no evidence of prior exposure to $M$. bovis.

${ }^{2}$ Comparative cervical tuberculin skin test after 180 days of comingling.

${ }^{3} \mathrm{Feces}$ and oronasal swab samples collected prior to comingling and on days 14, 28, 42, 56, 89 and 179 days of comingling. Isolate (sampling day after comingling)

${ }^{4}$ Isolation of mycobacteria from tissues collected at necropsy after 180 days of comingling. Neg = no mycobacteria isolated.

${ }^{5}$ For bacteriological isolation of mycobacteria, tissues were allocated into one of three pools; head (mandibular, parotid and medial retropharyngeal lymph nodes), thoracic (lung, mediastinal and tracheobronchial lymph nodes), or abdominal (mesenteric and hepatic lymph nodes).

${ }^{6}$ Unspeciated mycobacteria later identified by PCR as M. bovis BCG Danish.

${ }^{7}$ Deer died due to trauma unrelated to the experimental protocol prior to end of study.

UIM = Unspeciated mycobacteria found not to be M. bovis BCG Danish by PCR.

$\mathrm{NA}=$ not applicable

$\mathrm{ND}=$ not done.

Table 2: Summary of intradermal tuberculin test and bacteriological culture results from Mycobacterium bovis BCG Danish vaccinated deer co-mingled with unvaccinated deer for 180 days.

was used to simulate a commonly observed form of deer to cattle contact. Admittedly, it is not possible to rule out the potential for more intimate contact to occur between deer and cattle in a natural setting rather than the restrictive indirect contact allowed in the present study. However, one study done in the natural surroundings of northeastern Michigan showed direct deer-cattle interactions to be exceedingly rare; conversely, indirect contact was common [21].

As previously observed white - tailed deer are exposed to and harbor a wide variety of non-tuberculous mycobacteria $[22,23]$. Sensitivity to non-tuberculous mycobacteria may result in false positive results upon intradermal tuberculin testing. In the present study, to differentiate intradermal tuberculin reactions due to nontuberculous mycobacterial sensitivity from true $M$. bovis infection, the CCT was used in cattle and deer. The addition of $M$. avium PPD increases specificity of the CCT as compared to a single cervical test (SCT) using only $M$. bovis PPD. Deer or cattle sensitized to nontuberculous mycobacteria may be positive using a SCT; however, by using the CCT these sensitized animals can often be identified by reason of a greater change in skin thickness at the $M$. avium PPD injection site compared to the change in skin thickness at the $M$. bovis PPD injection site. In cattle, an IFN- $\gamma$ release assay is also available and may be used in place of the CCT. Unfortunately, efforts to create a similar IFN- $\gamma$ assay for deer have shown such an assay to be unreliable [24], leaving intradermal tuberculin testing the only means of antemortem diagnosis of exposure of $M$. bovis (including $M$. bovis $\mathrm{BCG})$ in deer.

Shedding of BCG from vaccinated deer to non-vaccinated deer has been documented previously $[7,23]$. To date, studies have not evaluated the mechanism of spread. In the current study, BCG was not isolated from feces of vaccinated deer and rarely from oronasal swabs of vaccinated deer. Proof of shedding is evident in the conversion of 4 out of 10 non-vaccinated deer from non-reactor to reactor status as identified by the CCT. Moreover, shedding is further evidenced by isolation of BCG from a non-vaccinated deer. Although transmission of BCG did occur, it is not possible, from the present study, to determine if deer to deer transmission occurred through direct or indirect contact.

Few studies have examined post-vaccinal spread of BCG in animals or humans. Post-vaccinal spread of BCG in humans is generally limited to shedding of BCG from ulcerated lesions at the site of vaccination. In one study 47 of 48 healthy, immunocompetent, volunteers vaccinated SC with BCG Connaught or Tice strains developed ulcerated lesions at the vaccination site. Viable BCG could be isolated from lesion exudate for up to 4 weeks [25]. Interestingly, similar to results in the present study, biopsies of ulcerated vaccination sites revealed granulomas, but no acid-fast bacteria [25].

Shedding has been evaluated in other species of wildlife. Shedding of virulent $M$. bovis has been documented in naturally infected badgers, [26] however; shedding of BCG is not seen in BCG vaccinated European badgers (Meles meles) after SC or intramuscular vaccination [27]. Moreover, unvaccinated badgers housed in the same enclosure, with ample opportunity for direct contact, did not become infected with BCG, or show immunologic responses suggestive of exposure to BCG. Oral vaccination of brushtail possums (Trichosurus vulpecula) with BCG showed fecal shedding of vaccine for 7 days after vaccination [28]. The magnitude of shedding was found to be 
Citation: Palmer MV, Thacker TC, Waters WR, Robbe-Austerman S, Harris BN (2010) Investigations on Deer to Deer and Deer to Cattle Transmission of the Vaccine Mycobacterium bovis Bacillus Calmette-Guérin (BCG). J Vaccines Vaccin 1: 104. doi:10.4172/2157-7560.1000104

low, with mean peak shedding of $1 \times 10^{4} \mathrm{CFU} / \mathrm{gm}$ feces 2 days after vaccination. In spite of inconsistent and transient shedding, BCG was isolated from mesenteric lymphoid tissue up to 8 weeks after vaccination [28]. In general, studies of BCG vaccinated wildlife have concluded that the risk for transmission of BCG from vaccinated wildlife to cattle is low; especially shedding sufficient to induce false positive tuberculin skin test reactions in cattle $[27,28]$.

The current study demonstrates that while BCG Danish may be shed and transmitted in a limited fashion from vaccinated to unvaccinated deer, the risk of transmission to cattle through indirect contact is low. The significance of BCG shedding from vaccinated deer is not trivial. Guidelines for the assessment of live biological agents suggest that persistence and shedding must be considered if the vaccine is shed as a live agent [29]. Moreover, if the agent is shed, the magnitude and duration of shedding should be investigated. Before implementation of a wildlife vaccination policy, further studies and both vaccine safety and efficacy will be required.

\section{Acknowledgements}

The authors thank Rebecca Jepsen, Theresa Anspach, Jason Crabtree, Jay Steffen and Doug Ewing for animal care and Bart Olthoff, Jessica Pollock, Rachel Huegel, Mike Howard, Shelly Zimmerman, Emma Frimml-Morgan and Allen Jensen for technical assistance. Mention of trade names or commercial products in this article is solely for the purpose of providing specific information and does not imply recommendation or endorsement by the U.S. Department of Agriculture.

\section{References}

1. Daszak $P$, Cunningham AA, Hyatt AD (2000) Emerging infectious diseases of wildlife--threats to biodiversity and human health. Science 287: 443-449.

2. Miller R, Kaneene JB (2006) Evaluation of historical factors influencing the occurrence and distribution of Mycobacterium bovis infection among wildlife in Michigan. Am J Vet Res 67: 604-615.

3. Schmitt SM, Fitzgerald SD, Cooley TM, Bruning-Fann CS, Sullivan L, et al (1997) Bovine tuberculosis in free-ranging white-tailed deer from Michigan. $J$ Wildl Dis 33:749-758.

4. O'Brien DJ, Fitzgerald SD, Lyon TJ, Butler KL, Fierke JS, et al. (2001) Tuberculous lesions in free-ranging white-tailed deer in Michigan. J Wildl Dis 37: $608-613$

5. O'Brien DJ, Schmitt SM, Fierke JS, Hogle SA, Winterstein SR, et al. (2002) Epidemiology of Mycobacterium bovis in free-ranging white-tailed deer, Michigan, USA, 1995-2000. Prev Vet Med 54: 47-63.

6. O'Brien DJ, Schmitt SM, Fitzgerald SD, Berry DE, Hickling GJ (2006) Managing the wildlife reservoir of Mycobacterium bovis: the Michigan, USA, experience. Vet Microbiol 112: 313-323.

7. Palmer MV, Thacker TC, Waters WR (2007) Vaccination of white-tailed deer (Odocoileus virginianus) with Mycobacterium bovis bacillus Calmette Guerin. Vaccine 25: 6589-6597.

8. Nol P, Palmer MV, Waters WR, Aldwell FE, Buddle BM, et al. (2008) Efficacy of oral and parenteral routes of Mycobacterium bovis bacille Calmette-Guerin vaccination against experimental bovine tuberculosis in white-tailed deer (Odocoileus virginianus): A feasibility study. J Wildl Dis 44: 247-259.

9. Bolin CA, Whipple DL, Khanna KV, Risdahl JM, Peterson PK, et al. (1997) Infection of swine with Mycobacterium bovis as a model of human tuberculosis. J Infect Dis 176: 1559-1566.

10. Palmer MV, Waters WR, Thacker TC, Greenwald R, Esfandiari J, et al. (2006) Effects of different tuberculin skin-testing regimens on gamma interferon and antibody responses in cattle experimentally infected with Mycobacterium bovis. Clin Vaccine Immunol 13: 387-394.

11. Palmer MV, Waters WR, Whipple DL, Slaughter RE, Jones SL (2004) Evaluation of an in vitro blood-based assay to detect production of interferon-gamma by Mycobacterium bovis-infected white-tailed deer (Odocoileus virginianus). J Vet Diagn Invest 16: 17-21.

12. U.S. Department of Agriculture AaPHIS: (2005) Bovine tuberculosis eradication Uniform methods and rules, effective. APHIS 91-45-011, US Government Printing Office, Washington, DC.

13. Hines N, Payeur JB, Hoffman LJ (2006) Comparison of the recovery of Mycobacterium bovis isolates using the BACTEC MGIT 960 system, BACTEC 460 system, and Middlebrook $7 \mathrm{H} 10$ and $7 \mathrm{H} 11$ solid media. J Vet Diagn Invest 18: $243-250$

14. Palmer MV, Waters WR, Whipple DL (2002) Susceptibility of raccoons (Procyon lotor) to infection with Mycobacterium bovis. J Wildl Dis 38: 266-274.

15. Palmer MV, Waters WR, Whipple DL (2002) Lesion development in white-tailed deer (Odocoileus virginianus) experimentally infected with Mycobacterium bovis. Vet Pathol 39: 334-340.

16. Kierschner P, Bottger EC (1998) Species identification of Mycobacteria using rDNA sequencing. Methods in Molecular Biology, Mycobacteria protocols. T. Parish and N. G. Stoker. Humana Press Inc., Totowa, NJ 101: 349-361.

17. Harmsen D, Dostal S, Roth A, Niemann S, Rothganger J, Sammeth M, Albert J, Frosch M, Richter E (2003) RIDOM: comprehensive and public sequence database for identification of Mycobacterium species. BMC Infect Dis 3:26.

18. Eisenach KD, Cave MD, Bates JH, Crawford JT (1990) Polymerase chain reaction amplification of a repetitive DNA sequence specific for Mycobacterium tuberculosis. J Infect Dis. 161: 977-981.

19. Roth A, Reischl U, Streubel A, Naumann L, Kroppenstedt RM, et al. (2000) Novel diagnostic algorithm for identification of mycobacteria using genusspecific amplification of the 16S-23S rRNA gene spacer and restriction endonucleases. J Clin Microbiol 38: 1094-1104.

20. Buddle BM, Aldwell FE, Skinner MA, de Lisle GW, Denis M, et al. (2005) Effect of oral vaccination of cattle with lipid-formulated BCG on immune responses and protection against bovine tuberculosis. Vaccine 23: 3581-3589.

21. Hill JA (2005) Wildlife-cattle interactions in Northern Michigan: Implications for the transmission of bovine tuberculosis. MS. Forest, Range, and Wildlife Sciences, Utah State University, Logan, Utah.

22. Palmer $M$, Thacker $T$, Waters $W$, Robbe-Austerman $S$, Lebepe-Mazur $S$, et al (2010) Persistence of Mycobacterium bovis Bacillus Calmette-Geurin (BCG) in white-tailed deer (Odocoileus virginianus) after oral or parenteral vaccination. Zoonoses Public Health [Epub ahead of print].

23. Palmer MV, Thacker TC, Waters WR (2009) Vaccination with Mycobacterium bovis BCG strains Danish and Pasteur in white-tailed deer (Odocoileus virginianus) experimentally challenged with Mycobacterium bovis. Zoonoses Public Health 56: 243-251.

24. Waters WR, Palmer MV, Thacker TC, Orloski K, Nol P, et al. (2008) Blood culture and stimulation conditions for the diagnosis of tuberculosis in cervids by the Cervigam assay. Vet Rec 162: 203-208.

25. Hoft DF, Leonardi C, Milligan T, Nahass GT, Kemp B, et al. (1999) Clinica reactogenicity of intradermal bacille Calmette-Guerin vaccination. Clin Infect Dis 28: $785-790$.

26. Clifton-Hadley RS, Wilesmith JW, Stuart FA (1993) Mycobacterium bovis in the European badger (Meles meles): epidemiological findings in tuberculous badgers from a naturally infected population. Epidemiol Infect 111: 9-19.

27. Lesellier S, Palmer S, Dalley DJ, Dave D, Johnson L, et al. (2006) The safety and immunogenicity of Bacillus Calmette-Guerin (BCG) vaccine in European badgers (Meles meles). Vet Immunol Immunopathol 112: 24-37.

28. Wedlock DN, Aldwell FE, Keen D, Skinner MA, Buddle BM (2005) Ora vaccination of brushtail possums (Tichosurus vulpecula) with BCG: immune responses, persistence of BCG in lymphoid organs and excretion in faeces. $N$ $Z$ Vet J 53: 301-306.

29. Sutton $A$ (2008) Environmental assessment requirements for live biological drugs. Clin Infect Dis 46: 112-151. 